Präv Gesundheitsf 2022 · 17:96-103 https://doi.org/10.1007/s11553-021-00841-0 Eingegangen: 17. Dezember 2020

Angenommen: 2. März 2021

Online publiziert: 25. März 2021

(c) Der/die Autor(en) 2021

Yvonne Adam • Eva-Maria Berens ${ }^{1}$

${ }^{1}$ Interdisziplinäres Zentrum für Gesundheitskompetenzforschung (IZGK), Universität Bielefeld, Bielefeld, Deutschland

\title{
Gesundheitskompetenz für die Familie: Frauen mit Migrationshintergrund als Mittlerinnen
}

\author{
Eine qualitative Analyse von \\ Fokusgruppendiskussionen und \\ Einzelinterviews
}

der Informationsverarbeitung: Suchen/ Finden, Verstehen, Beurteilen und Anwenden. Dabei stehen die Fähigkeiten des Individuums und die Anforderungen des Gesundheitssystems in Wechselwirkung. Auch gesellschaftliche, ökologische und situative Determinanten beeinflussen die Gesundheitskompetenz [22, 26].

Insgesamt hat mehr als die Hälfte der Bevölkerung in Deutschland eine geringe Gesundheitskompetenz und sieht sich somit vor große Schwierigkeiten im Umgang mit Gesundheitsinformationen gestellt $[27,28]$. Dies ist besonders beachtenswert, da sich die Gesundheitskompetenz unmittelbar auf die gesundheitliche Situation auswirkt: Menschen mit hoher Gesundheitskompetenz ernähren sich beispielsweise besser, bewegen sich häufiger und fühlen sich insgesamt gesünder [15, 27, 28]. Aus der Perspektive der Gesundheitsförderung wird Gesundheitskompetenz daher als Ressource für den Erhalt und die Verbesserung der individuellen Gesundheit und auch der lebensweltlich bezogenen gesundheitsbeeinflussenden Faktoren verstanden [1]. 
Tab. 1 Sample Fokusgruppen $(F K)$ und

Einzelintreviews (EI)

Sample Fokusgruppen

10 Frauen mit türkischsprachigem MH (FK türk. MH):

30-46 Jahre, Durchschnitt 34,5 Jahre

10 Frauen sind berufstätig

Bildungsabschlüsse: 2 Hochschulabschluss,

2 Abitur, 3 Realschule, 3 Hauptschule

10 Frauen mit russischsprachigem MH (FK russ. $\mathrm{MH})$ :

21-49 Jahre, Durchschnitt 38 Jahre

9 Frauen sind berufstätig, eine studiert

Bildungsabschlüsse: 5 Hochschulabschluss,

3 Abitur, 2 Realschule

Sample Einzelinterviews

6 Interviewpartnerinnen aus der migrantischen Selbsthilfe, 3 türkischsprachige (El türk. $\mathrm{MH}$ ) und 3 russischsprachige (El russ. $\mathrm{MH}$ ): 36-56 Jahre, Durchschnitt 44 Jahre

6 Frauen sind berufstätig

Bildungsabschlüsse: 4 Hochschulabschluss,

2 Realschule

MH Migrationshintergrund

geringere Gesundheitskompetenz. Eine differenziertere Betrachtung ist notwendig, die für Deutschland jedoch noch weitestgehend aussteht.

In der Sozialforschung werden Migrationsfamilien meist mit Bildungsferne, Traditionalismus oder Identitätskonflikten assoziiert und damit als bedürftig eingestuft. Die Defizitorientierung prägt bislang auch das Bild von Menschen mit $\mathrm{MH}$ als vulnerable Gruppe in der Gesundheitskompetenzforschung.

\section{Family Health Literacy}

Die Gesundheitskompetenz im Kontext der Familie wurde bislang wenig beachtet. Forschung dazu bündelt sich unter dem Konzept der Family Health Literacy. Es basiert auf einer sozial geteilten Gesundheitskompetenz [10, 21]. Wissen über Gesundheit wird nicht als individuelle Errungenschaft verstanden, sondern als lokal erworben und in sozialen Beziehungen mitproduziert. Gesundheitskompetenz als soziale Praxis ist folglich situativ, dynamisch, mehrdimensional und eingebettet in soziale Netzwerke [25]. Bisher konzentrierte sich die Forschung auf die Familie als primäre Sozialisationsinstanz, die maßgeblich das spätere Gesundheitsverhalten der Kinder bestimmt [6, 13, 19]. Einzelne interna- tionale Untersuchungen zeigen jedoch, dass Erwachsene die Funktion von Gesundheitsberater*innen für die gesamte Familie einnehmen [8, 29].

Im Folgenden wird nicht die Rolle der Gesundheitskompetenz der Eltern für ihre Kinder untersucht, sondern die der erwachsenen Kinder für ihre Eltern. Mit einem qualitativen Ansatz wird die Perspektive von Frauen mit $\mathrm{MH}$ erhoben, da sie im Kontext von Gesundheitskompetenz eine wichtige Rolle für die gesamte Familie einzunehmen scheinen. So wurden bereits vor 17 Jahren Frauen als zuständig für die Gesundheit der Familie beschrieben [34]. Auch in einer aktuellen Studie wird zitiert, dass Frauen mit $\mathrm{MH}$ sich erst dann selbst gesund fühlen, wenn es der gesamten Familie gut geht [6]. Dies ist ein hoher Motivator, sich mit Gesundheitsinformationen auseinander zu setzen.

In der vorliegenden Untersuchung stehen Frauen der zweiten Migrationsgeneration $^{2}$ im Mittelpunkt, da sie sich häufig nicht

nur um die Kinder, sondern auch um die älteren Familienmitglieder kümmern und aufgrund ihrer Rolle als Sprach- und Kulturmittler*innen über Informationsbedürfnisse der zugewanderten ersten Generation Auskunft geben können.

Um einen ressourcenorientierten Blick einnehmen zu können, wurden die Erfahrungen gut deutschsprechender, berufstätiger Frauen mit MH ausgewertet.

\section{Ziel und Methodik}

Im Fokus der vorliegenden Auswertung stehen Erfahrungen von türkisch- und russischsprachigen Frauen der zweiten Migrationsgeneration im frühen und mittleren Erwachsenenalter. Das Ziel liegt darin, Erkenntnisse zu ihrem Umgang mit gesundheitsrelevanten In-

\footnotetext{
2 Zur zweiten Migrationsgeneration werden die nachgeholten bzw. die in Deutschland geborenen Kinder der ersten Generation gezählt, deren Kinder entsprechend zur dritten Generation (vgl. [36]). In diesem Beitrag wird der alltagssprachliche Begriff "mittlere“ Generation verwendet, um die Situation der teilnehmenden Frauen zwischen der Versorgung der Kinder und derVersorgung der Eltern auszudrücken.
}

formationen zu gewinnen: Welche Wege beschreiten sie, um Informationen $\mathrm{zu}$ suchen und welche Herausforderungen gibt es beim Verstehen, Einschätzen und Nutzen von Informationen. Dafür wurden zwei Fokusgruppendiskussionen mit je 10 Teilnehmerinnen und ergänzend sechs Einzelinterviews mit Frauen aus der migrantischen, gesundheitsbezogenen Selbsthilfe durchgeführt (vgl. - Tab. 1). Fokusgruppendiskussionen eignen sich besonders, um unterschiedliche Perspektiven von Menschen auf ihre Erfahrungen zu sammeln, da die Teilnehmenden sich in der Diskussion gegenseitig zu Reflektionen, Kontrastierungen und Einordnungen anregen können. Sind die Gruppen nach einem bestimmten Kriterium wie beispielsweise „Herkunftsland“ bzw. „Sprachgruppen“ zusammengestellt, können auch kollektive Orientierungsmuster thematisiert werden. Dabei gilt es zu beachten, dass die Ergebnisse von Fokusgruppenstudien nicht repräsentativ sind, aber einen intensiven Einblick in Denkweisen und Handlungsprozesse geben können [7, 11].

Die Leiterinnen von gesundheitsbezogenen Selbsthilfegruppen für Migrant*innen wurden in Einzelinterviews befragt, weil sie selbst die Erfahrungen im Umgang mit Gesundheitsinformationen der Frauen mittleren Alters der Fokusgruppen teilen und weil sie Ansprechpartnerinnen für Menschen der gleichen Sprachgruppe sind und somit über ein breites Wissen über die Probleme beim Finden, Verstehen, Beurteilen und Anwenden von Informationen verfügen.

Die Sprachen Türkisch und Russisch wurden ausgewählt, da diese in Haushalten von Menschen mit MH in Deutschland am häufigsten vertreten sind. Alle teilnehmenden Frauen sind entweder selbst in Deutschland geboren oder leben schon seit der Kindheit bzw. Jugend hier und sprechen sehr gut Deutsch.

Alle Fokusgruppenteilnehmerinnen und Interviewpartnerinnen wurden $\mathrm{zu}$ Beginn über die Ziele der Studie informiert und über die Anonymisierung der Daten aufgeklärt. Eine Einwilligung zur Aufzeichnung und Verwendung der Daten wurde eingeholt. Die Diskussionen 
und Interviews wurden aufgezeichnet und nach einfachen Transkriptionsregeln in Schriftform gebracht. Die Teilnehmerinnen erhielten ein Pseudonym mit zufällig gewählten türkischen bzw. russischen Vornamen [9]. Die Erstautorin verfasste jeweils ein reflektierendes Gedächtnisprotokoll [14].

Der Leitfaden beinhaltete folgende Themen:

- Verständnis von Gesundheitskompetenz,

- Erfahrungen beim Suchen und Finden von Gesundheitsinformationen und Orientierung im Gesundheitssystem,

- Verstehen von Gesundheitsinformationen,

- Beurteilen und Einschätzen von Gesundheitsinformationen sowie Vertrauen in Gesundheitsinformationen,

- Entscheidungsfindung zum Anwenden von Gesundheitsinformationen,

- Informationsquellen,

- Wünsche zur Verbesserung.

Im Einzelinterview wurden die Frauen zusätzlich gebeten, zu den Ergebnissen der Fokusgruppendiskussionen Stellung zu nehmen.

Es wurde eine qualitative Inhaltsanalyse nach Kuckartz und Mayring durchgeführt [16,20]. Dafür wurde das gesamte Material in die Software MAXQDA eingepflegt. Die Kategorien Informationen suchen, verstehen, beurteilen und anwenden entlang der vier Schritte der Informationsverarbeitung [30] wurden deduktiv kodiert. Die intergenerationalen Themen haben die teilnehmenden Frauen selbst eingebracht. Dieses Vorgehen ermöglicht es, sowohl allgemeine Aussagen zur Informationsverarbeitung zu erhalten und gleichzeitig migrationsspezifische Besonderheiten herauszuarbeiten.

\section{Ergebnisse}

Die türkisch- und russischsprachigen Frauen der mittleren Generation zeigen sich insgesamt versiert im Umgang mit Gesundheitsinformationen und setzen ihre Mehrsprachigkeit bewusst ein. Im Folgenden wird entlang der vier Schritte

Präv Gesundheitsf 2022 · 17:96-103 https://doi.org/10.1007/s11553-021-00841-0

(c) Der/die Autor(en) 2021

Y. Adam · E.-M. Berens

\section{Gesundheitskompetenz für die Familie: Frauen mit Migrationshintergrund als Mittlerinnen. Eine qualitative Analyse von Fokusgruppendiskussionen und Einzelinterviews}

\section{Zusammenfassung}

Hintergrund. Menschen mit Migrationshintergrund gelten als vulnerable Gruppe, deren Gesundheitskompetenz gefördert werden muss. Bisher gibt es noch wenige Studien, die neben dem Migrationshintergrund auch den gender- und intergenerationalen Einfluss untersuchen.

Ziel und Methodik. Entlang der vier Schritte der Informationsverarbeitung Suchen, Verstehen, Beurteilen und Anwenden werden zwei Fokusgruppen und sechs Einzelinterviews mit türkisch- und russischsprachigen Frauen der mittleren Generation analysiert, um aufzuzeigen, welchen Einfluss die Gesundheitskompetenz der Frauen auf die gesamte Familie hat.

Ergebnisse. Die Frauen setzen ihr Wissen, ihre Fähigkeiten und insbesondere ihre Mehrsprachigkeit gezielt ein, um ihre älteren Angehörigen zu unterstützen. Beim Suchen,
Verstehen und Beurteilen von Informationen wird deutlich, dass Entscheidungen in der Familie diskutiert und vom sozialen Umfeld beeinflusst werden. Beim Anwenden motivieren die Frauen ihre älteren Angehörigen zu einem gesundheitsförderlichen Verhalten. Schlussfolgerung. Die Frauen treten als Mittlerinnen für die Gesundheit der gesamten Familie auf. Über sie könnten auch schwer erreichbare Gruppen differenz- und ressourcenorientiert gefördert werden. Für die Konzeption einer Family Health Literacy ist nicht nur der Einfluss von Eltern auf ihre Kinder interessant, sondern auch von erwachsenen Kindern auf ihre Eltern.

Schlüsselwörter

Gesundheitsinformation · Qualitativ · Health Literacy · Assoziation · Familie

\section{Health literacy for the family: Women with a migration background as mediators. A qualitative analysis of focus group discussions and individual interviews}

Abstract

Background. People with a migration background are considered a vulnerable group whose health literacy needs to be strengthened. So far, there are only a few studies that have investigated gender and intergenerational aspects in addition to the migration background.

Objectives and methodology. Two focus groups and six individual interviews with Turkish-speaking and Russian-speaking middle-generation women are analyzed along the four steps information processing searching, understanding, assessing and applying in order to show the impact that the women's health literacy has on the entire family.

Results. The women use their knowledge, their competences and especially their multilingualism in a targeted manner to support their elderly relatives. When looking for, understanding and judging information, it becomes clear that decisions are discussed in the family and are influenced by the social environment. When using it, women motivate their older relatives to behave in a healthpromoting manner.

Conclusion. Women act as mediators for the health of the entire family. Through them, groups that are difficult to reach could also be strengthened in a difference- and resource-oriented manner. In relation with family health literacy, not only the influence of parents on their children is interesting, but also adult children's impact on their parents.

Keywords

Health information $\cdot$ Qualitative $\cdot$ Health literacy · Association · Family 
der Informationsverarbeitung herausgestellt, vor welche Herausforderungen sich Frauen der mittleren Migrationsgeneration mit Gesundheitsinformationen gestellt sehen und welche Ressourcen sie dabei einbringen.

\section{Informationen für die Familie suchen}

Die Entscheidung, mit einer Informationssuche zu beginnen, wird meist anlassbezogen oder sogar aus einer Notlage heraus gefällt, z. B. wenn die Eltern nicht mehr zu Hause gepflegt werden können. Die Suche selbst gehen die Frauen dann selbstbewusst an: Man müsse nur gut recherchieren, dann wäre alles $\mathrm{zu}$ finden (Fedora, russ. MH: 117). Eine Einrichtung wie einen Pflegedienst suchen die Frauen im Internet oder sie bekommen Informationsmaterial im Stadtteil ausgehändigt (FK türk. MH: 137-140). Diese proaktive Informationssuche kostet die Frauen aber auch viel Energie und Kraft (Inci, türk. MH: 55). Denn sie wird dadurch komplex, dass eine erhaltene Information den Bedarf nach weiteren Informationen auslöst und viele verschiedene Quellen gesichtet werden (müssen). Dazu gehört bei den Frauen mit $\mathrm{MH}$ auch, parallel muttersprachliche Informationen einzuholen.

Es beginnt schon damit, es gibt das, aber wie bekomme ich diesen Anspruch? Dann geht es weiter, dann braucht die zu plegende Person einen Pflegegrad, wie bekomme ich diesen Pflegegrad? Dann kommt der MDK ins Spiel. Wie läuft das? Das sind solche Dinge, also dieser rote Faden, das ist total schwierig. Ich habe mich wie so ein Jäger und Sammler gefühlt, ich musste die Krankenkasse anrufen, mit den Ärzten in Kontakt treten, ... habe mich mit meiner Tante ausgetauscht, viel auf Arbeit unterhalten, dann noch diesem und jenen erfahren, es gibt noch diese Möglichkeit. Auch diese Zusatz-Geschichten wie bei Diabetes mit der Podologie, dass das auch allen zusteht, aber es gibt grundsätzlich Sachen, das weiß auch nicht der Hausarzt. (Inci, türk. MH: 144)

In dem Zitat wird deutlich, dass die Gespräche mit Verwandten, Bekannten oder Kolleg*innen entscheidend dafür sind, neue Möglichkeiten der Informationssuche - auch im Herkunftsland - in Betracht zu ziehen. Eine einzelne Informationsquelle, wie hier z. B. der Hausarzt oder die Hausärztin, kann nicht über alle Optionen Bescheid wissen. Je mehr eine Person im Gespräch mit anderen bleibt, je mehr Informationen erhält sie. Dies unterstreicht den situativen und sozialen Charakter der Informationssuche: Sie wird mehr oder weniger zufällig gelenkt durch Personen aus dem Lebensumfeld, die ihr Wissen beisteuern. Die Initiative zur Informationssuche wird von einem Individuum ergriffen, der weitere Verlauf der Suche dann aber von vielen Personen - professionellen und Laien - mitbestimmt. Die Mehrsprachigkeit spielt bei der Suche zudem eine wichtige Rolle, Informationen werden häufig nicht nur auf Deutsch, sondern auch der Muttersprache recherchiert, wie beim Verstehen noch näher erläutert wird. Dies unterstreicht die Komplexität der Informationssuche.

Es verwundert nicht, dass die Frauen sich wünschen, dass das Internet in leichter und in mehreren Sprachen sein soll, „das brauchen wir hier alle“, stellt eine Interviewpartnerin fest (Azra, EI türk. $\mathrm{MH}$ : 76). Sie erhoffen sich zudem eine Instanz wie mehrsprachige Infotelefone oder Beratungsstellen, die die Weichen in der Informationsflut stellt und verhindert, sich in den vielzähligen Angeboten des Gesundheitssystems zu verlieren (Adile türk. MH: 193, Rabia, türk. MH: 150; 195).

\section{Informationen für die Familie beurteilen}

Ihre eigene Bildung und ihr erworbenes (Fach-)Wissen sind für die Frauen die Grundlage, Informationen einzuschätzen und zu beurteilen. Sie lesen zu bestimmten Fachthemen, z. B. zu Impfungen für ihre Kinder (Jelena, EI russ. MH: 3) oder zu Wirkungen von Medikamenten für ihre Eltern (Inci, türk. MH: 133). Dabei vertrauen sie wissenschaftlichen Beiträgen mit Quellennachweisen (z. B. Adile, türk. MH: 82; Anastasija, russ. $\mathrm{MH}: 81$ ), sind aber darüber hinaus überwiegend ratlos, wo zuverlässige Information $\mathrm{zu}$ erhalten ist (FK russ. MH: 155).
Deshalb wünschen sich die Frauen eine Kontrollinstanz (Slata, russ. MH: 152).

Eine weitere Strategie ist für die Frauen, Informationen aus mehreren Quellen zusammenzutragen und sie vergleichend $\mathrm{zu}$ prüfen. Deshalb ist Information aus verschiedenen Perspektiven für sie wichtig.

Ich suche dann Informationen im Internet, sehr viel und dann frage ich meine Ärzte, unterschiedliche Ärzte, was sie davon halten.... Und da versuche ich halt überall so ein bisschen Input zu kriegen und dann selber auszuwerten und zu analysieren. (Fedora, russ. MH: 30)

Grundsätzlich haben die Frauen die Haltung, dass nur sie selbst für sich und ihre Angehörigen beurteilen können, welche Informationen die passenden sind. „Das ist ganz wichtig, dass wir für uns selbst entscheiden, was ist für uns gut", meint eine Interviewpartnerin (Elif, EI türk. MH: 47). Für die Beurteilung, welche Information "gut" ist, wird dann wieder das soziale Umfeld bemüht. Bei den Pflegediensten werden beispielsweise die unterschiedlichen Einrichtungen nach Hörensagen bewertet. Neben der fachlichen Information prägt folglich der lebensweltliche Kontext der Familie die Einschätzung der Gesundheitsdienstleistung.

\section{Informationen für die Familie verstehen}

Beim Verstehen von Gesundheitsinformationen ist eine ausgeprägte Sprachkompetenz von großer Bedeutung, denn die Eltern und andere Angehörige mit geringeren Deutschkenntnissen werden gezielt unterstützt. Die Frauen begleiten ihre Angehörigen und helfen dabei, die Aussagen des Gesundheitspersonals zu verstehen. Gesundheit bzw. Krankheit wird als ein Thema eingeschätzt, das sensibel ist und über das besser in der Muttersprache gesprochen wird, da es auch von existentiellen Ängsten begleitet sein kann (Nadia, russ. MH: 124). Neben der Funktion als Übersetzerin, sind die Frauen also auch eine emotionale Stütze für ihre Angehörigen.

Darüber hinaus sind sie auch Kulturmittlerinnen. Dies wird deutlich 
in der Fokusgruppendiskussion über die Konsequenzen, wenn mangelnde Deutschkenntnisse oder kulturelle Hintergründe es erschweren, sich verständlich auszudrücken. Besonders in der Kommunikation zwischen Ärzt*innen und Patient*innen sehen die Frauen bei interkulturellen Missverständnissen gravierende Folgen: Wenn Ärzt*innen die Beschreibungen der Beschwerden ihrer Patient ${ }^{*}$ innen nicht verstehen, kann dies im schlimmsten Falle zu einer Fehlbehandlung führen (Nadia, russ. MH: 122).

Die Angst, sich nicht verständlich machen zu können bzw. nicht verstanden $\mathrm{zu}$ werden, halte ihre Landsleute sogar oft davon ab, Hilfe einzufordern, so die Einschätzung der russischsprachigen Interviewpartnerinnen. Sie hätten Angst, etwas falsch $\mathrm{zu}$ machen oder nur allgemeine Informationen zu erhalten, die nicht zu ihrer persönlichen Situation passen (Irina, EI russ. MH: 24, Jelena, EI russ. $\mathrm{MH}: 33)$.

Die Übersetzungsarbeit bei einem Arztbesuch mit Angehörigen kann dann weit über das wörtliche Dolmetschen hinausgehen. Die Frauen erklären und interpretieren fehlende Begriffe oder Fremdworte und medizinische Untersuchungen so, dass sie für Adressat*innen verstehbar sind (Nadja, russ. MH: 129; Esin, türk. MH: 115). Besonders schwierig ist dies, wenn es keine begriffliche Entsprechung in der Muttersprache gibt und eine Situation doppelter Sprachlosigkeit entsteht: Den Jüngeren fehlen die "richtigen“ Worte zum Übersetzen und den Älteren das Verständnis dafür, was sich hinter den Begriffen verbirgt. Beispielsweise berichten türkischsprachige Teilnehmerinnen, wie schwierig es sei, über Depression und Demenz in geeigneten Worten zu sprechen. Der Bedeutungszusammenhang im Türkischen sei negativ und mit persönlicher Schwäche assoziiert, so dass mit Scham verbunden sei, von diesen Erkrankungen betroffen zu sein (Elif, EI türk. MH: 30). Teil der Übersetzungsarbeit ist es dann, Aufklärung zu leisten.

Wenn die Frauen Angehörige beim Arztbesuch begleiten, machen sie oft die Erfahrung, dass das medizinische Personal detaillierter informiert.
Ich habe auch das Gefühl ..., dass die Ärzte dann auch noch mehr erklären. Wenn sie merken, sie ist alleine, dann wird es ganz kurzgehalten und wenn sie merken, da ist jemand, der perfekt Deutsch sprechen kann, dann erzählen sie viel detaillierter. (Rabia, türk. MH: 116)

Offensichtlich hat geringe Sprachkenntnis zur Folge, dass keine ausführlichen Erklärungen gegeben werden, da im Vorhinein ein Nicht-Verstehen unterstellt wird. Das ist deshalb paradox, weil gerade durch fehlende Sprachkenntnisse ein größerer Bedarf an Erklärung, Kontextinformation und Interpretation besteht. Dies unterstreicht die wichtige Funktion der Frauen der mittleren Generation, die diese Lücke füllen.

Informationen anwenden: gesundheitsbezogene Entscheidungen der Familie unterstützen und gesundheitsförderliches Verhalten forcieren

Wie bereits beim Beurteilen von Informationen sehen die Frauen auch in der Anwendung einen Zusammenhang mit vorhandenen literalen Kompetenzen und den gegebenen lebensweltlichen Bedingungen. Die Menschen der ersten Generation oder mit einer geringen Schulbildung benötigten eine persönliche Ansprache, um Informationen umsetzen zu können (Azra, EI türk. MH: 74). Am Beispiel der für die Frauen wichtigen Themen Ernährung und Bewegung wird deutlich, wie sie ihr eigenes Wissen und ihre Vorstellungen eines gesundheitsförderlichen Verhaltens für die Familie einsetzen.

In der Fokusgruppe mit türkischsprachigem Hintergrund wird reflektiert, dass die Gastarbeiter ${ }^{*}$ innengeneration früher in der ländlichen Türkei gesünder gelebt hätte und die Ernährung durch die selbst produzierten Lebensmittel ganz anders gewesen sei. Da viele keine gute Bildung hätten und somit über weniger Wissen über einen gesunden Lebensstil unter den veränderten Lebens- und Arbeitsbedingungen in Deutschland verfügten, würden sie sich hier nicht gut ernähren und nicht ausreichend bewegen (FK türk. MH: 103-113). Die Frauen sehen es des- halb als ihre Aufgabe an, entsprechende Informationen weiterzugeben und Vorschläge zu unterbreiten, was für eine gute Gesundheit zu tun ist (Nuran, türk. MH: 88). Eine Teilnehmerin berichtet beispielsweise, wie sie der traditionell eingestellten Großmutter ihres Mannes beibringen wollte, sich gesund zu ernähren (Hülja, türk. MH: 108). Solche praktischen Vorschläge für ein besseres $\mathrm{Ge}$ sundheitshandeln sind aber nicht nur an Bildungsunterschiede gebunden. Darauf weist eine Diskutantin hin, deren Eltern ebenso wie sie selbst studierten und beschreibt, wie sie ihnen eine Lauf-App auf deren Smartphones geladen habe, damit sie jeden Tag 1000 Schritte gehen.

Ich finde das kommt auch von unserer Generation, dass man sie so von hinten anschiebt und sagt, los mach mal! (Esin, türk. MH: 109)

Die Frauen sind also der Ansicht, dass (erwachsene) Kinder ihren Eltern etwas beibringen können. Informationen über ein gutes Gesundheitsverhalten werden zwischen den Generationen ausgetauscht. Gesundheitshandeln erweist sich folglich als dynamisch und ist nicht allein von der Gesundheitskompetenz der betreffenden Person abhängig.

\section{Zusammenfassung und Diskussion}

Die Analyse der Fokusgruppendiskussionen und der Einzelinterviews hat gezeigt, dass die türkisch- und russischsprachigen Frauen der mittleren Generation sich für die Gesundheit ihrer Angehörigen verantwortlich fühlen und auch für ihre Eltern gesundheitsbezogene Informationen verarbeiten und Entscheidungen den Weg ebnen. Sie treten als Managerinnen der Gesundheit der Familien auf und berufen sich dabei auf ihr eigenes (Fach-)Wissen, auf ihre Gesundheitssystem- und ihre Sprachkompetenz. Die vier Schritte der Informationsverarbeitung Suchen/Finden, Verstehen, Beurteilen und Anwenden gehen sie nicht nur für sich selbst, sondern sie machen Informationen auch für ihre Eltern sichtbar, verstehbar, einschätzbar und anwendbar.

Besonders der intergenerationale Charakter wird hierbei deutlich: Die 
jüngere Generation setzt ihre Gesundheitskompetenz ein, um die ältere Generation zu befähigen und auch aktiv eine Änderung des Gesundheitsverhaltens herbeizuführen. Im Migrationskontext ist dies von besonderer Wichtigkeit: Die mittlere Generation kennt die Lebenswelten sowohl in den Herkunftsländern als auch in Deutschland mit ihren gesundheitsbezogenen Rahmenbedingungen und kann hier - nicht nur sprachlich - vermittelnd tätig werden.

Das (muttersprachliche) soziale Umfeld ist bei allen vier Schritten unmittelbar beteiligt: Es kann die Pfade der Informationssuche lenken und den Radius vergrößern, es unterstützt beim Verstehen von Informationen und Meinungen werden zur Beurteilung und Anwendung eingebracht. Dies zeigt deutlich den situativen und sozialen Charakter von Gesundheitskompetenz.

Die Gruppe der Frauen mit $\mathrm{MH}$ ist in sich jedoch als heterogen zu betrachten ${ }^{3}$, wie die vorliegende Untersuchung abermals bestätigt. Sie unterscheiden sich nach Alter, Migrationserfahrung, Aufenthaltsdauer, ethnischer Zugehörigkeit, sozioökonomischen Ressourcen und individueller Biografie. So beziehen sich die Frauen mit türkischer Herkunft beispielsweise häufig auf die Bedingungen der Gastarbeiter ${ }^{*}$ innengeneration und die russischsprachigen Frauen auf die spezifischen Zustände der einzelnen postsowjetischen Länder. In der Gesundheitsforschung werden aber vorwiegend Frauen mit $\mathrm{MH}$ insgesamt adressiert und der vulnerablen Gruppe zugeordnet: Bisherige Untersuchungen weisen beispielsweise auf spezifische Gesundheitsrisiken und Zugangsbarrieren hin $[4,17,24]$. Die vorliegende Untersuchung lenkt den Blick auf die Heterogenität und weg von der Vulnerabilität hin zu einer

\footnotetext{
${ }^{3}$ Im Jahr 2019 haben von den Frauen mit Migrationshintergrund, die im Besitz der deutschen Staatsbürgerschaft sind, 2,7 Mio. "eigene Migrationserfahrung", sind also nach Deutschland eingewandert; 2,19 Mio. wurden dagegen in Deutschland geboren, haben also keine eigene Migrationserfahrung. Hinzu treten 4,1 Mio. Ausländerinnen, die nach Deutschland migriert sind und 711.000 Ausländerinnen, die seit ihrer Geburt in der Bundesrepublik leben (vgl.Statistisches Bundesamt [36]).
}

Ressourcen- und Potenzialorientierung, insbesondere in der mittleren Generation. Andere Untersuchungen stützen dies und zeigen, dass Frauen mit $\mathrm{MH}$ in der Familie Bildungserfolge fordern und fördern [31].

Die vorliegende Untersuchung bestätigt auch das Postulat, auf allen Ebenen eine Differenzorientierung gegenüber Menschen mit MH einzunehmen [6]. Die dargelegten Beispiele der Suche nach einem „guten“ Pflegeplatz für die Eltern oder einer „guten“ Ernährung zeigen, dass es keine allgemeingültige Vorstellung von "gut“ gibt, dies wird angesichts unterschiedlicher Konzepte von adäquater Pflege oder Gesundheit und Krankheit, von Geschlechterrollen, von unterschiedlichen Kommunikationsstrategien beispielsweise über psychische Erkrankungen und ebenso den sozioökonomischen Ressourcen der Familie deutlich. Vielmehr werden gesundheitsbezogene Entscheidungen entsprechend der spezifischen Begebenheiten gefällt. Dies bedarf einer Akzeptanz von Unterschiedlichkeit in den Vorstellungen einer ,guten Gesundheit“. Dabei gilt es, die besondere Rolle der Frauen der mittleren Generation zu würdigen, die versuchen, Konzepte ihrer älteren Angehörigen mit denen der von ihnen vielleicht im deutschen Gesundheitssystem erlernten auszupendeln und vermittelnd tätig zu werden.

Für die Konzeption einer Family Health Literacylassen sich aus dargestellter Studie folgende Anknüpfungspunkte ableiten: Aus der intergenerationalen Perspektive heraus erweitert sich der Fokus nicht wie bisher auf der Weitergabe der Gesundheitskompetenz von Eltern an ihre Kinder, sondern von erwachsenen Kindern an ihre Eltern. Gesundheitskompetenz in der Familie ist somit nicht eindimensional, sondern immer wechselseitig. Jedes Mitglied kann in seinem spezifischen Kontext Gesundheitsinformationen verarbeiten und dieses Wissen dann in die Familie einbringen. Auch die Genderperspektive unterstützt diese Mehrdimensionalität von Gesundheitskompetenz in der Familie. Die Migrationsperspektive lenkt den Blick auf einen erweiterten Familienbegriff: Die Frauen unterstützen nicht nur ihre unmittelbaren Angehörigen, sondern auch andere Personen aus der Community derselben Sprachgruppe. Zudem löst sie auf, Menschen mit $\mathrm{MH}$ als homogene Gruppe zu sehen. Die Frauen der mittleren Generation zeigen Stärke in der Gesundheitskompetenz für die Familie und gleichzeitig spezifische Unterstützungsbedarfe.

Für Interventionen zur Stärkung der Gesundheitskompetenz bedeutet dies, dass vorhandene Programme und Angebote, die sich bisher häufig alle Menschen mit MH bzw. Menschen aus spezifischen Herkunftsländern adressieren, sich stärker an Frauen mit MH der mittleren Generation richten sollten. Besonders schwer erreichbare Gruppen, wie beispielsweise Zugewanderte der ersten Generation mit geringen Deutschkenntnissen oder Geflüchtete können über die Frauen angesprochen werden. Eine intergenerationale Perspektive erlaubt auch, lebensweltlich orientierte Räume für die Vermittlung von Gesundheitswissen und Orte der Entscheidungsfindung ins Auge zu fassen. Wie die Frauen in der vorliegenden Studie ausgesagt haben, kann diese Mittlerinnenfunktion v. a. angesichts der Flut an Gesundheitsinformationen für sie selbst $\mathrm{zu}$ einer Überforderung werden, bis hin zu einer Gefährdung der eigenen Gesundheit. Sie gehören zwar in der Informationsverarbeitung nicht zur vulnerablen Gruppe der Menschen mit MH, dennoch benötigen sie als Mittlerinnen Unterstützung.

\section{Limitationen}

Für ein tiefergehendes Verständnis der geschlechtsspezifischen Rollen hinsichtlich der Gesundheitskompetenz in der Familie wäre weitere qualitative Forschung v.a. auch mit Männern von Nöten. Levin-Zamir et al. weisen z. B. darauf hin, dass in Pakistan Männer für die (finanzielle) Entscheidung zum Gesundheitshandeln zuständig sind, auch wenn die Frauen eine höhere Gesundheitskompetenz haben [18].

Viele der hier getroffenen Aussagen sind sicherlich auch für Frauen ohne MH gültig. Forschung wäre wünschenswert, die den Schwerpunkt auf die Stärkung der Gesundheitskompetenz aller Frauen der mittleren Generation legt. Diese müss- 
te auch Untersuchungen beinhalten $\mathrm{zu}$ Frauen, die nicht berufstätig sind oder sich durch weitere Variablen im sozioökonomischen Status unterscheiden.

\section{Fazit für die Praxis}

- Die Gruppe der Menschen mit Migrationshintergrund (MH) muss differenziert betrachtet werden.

- Frauen mit MH der mittleren Generation können als Mittlerinnen für schwer erreichbare Gruppen der Menschen mit MH adressiert werden.

- Statt einer Defizitperspektive sollte eine Ressourcenorientierung eingenommen werden.

- Gesundheitskompetenz wird in der Familie geteilt: Dies erfordert einen intergenerationalen Zugang.

- Die Familie ist ein wichtiges Setting, in dem Gesundheitskompetenz gestärkt werden kann.

- Frauen mit MH der mittleren Generation sind durch die Unterstützung ihrer Familien sehr beansprucht: Dies bedarf der Würdigung und geeigneter Maßnahmen, deren Gesundheit nicht aus dem Blick zu verlieren.

\section{Korrespondenzadresse}

\section{Dr. Eva-Maria Berens}

Interdisziplinäres Zentrum für Gesundheitskompetenzforschung (IZGK), Universität Bielefeld

Universitätsstraße 25, 33615 Bielefeld, Deutschland

eva-maria.berens@uni-bielefeld.de

Funding. Open Access funding enabled and organized by Projekt DEAL.

\section{Einhaltung ethischer Richtlinien}

Interessenkonflikt. Y. Adam und E.-M. Berens geben an, dass kein Interessenkonflikt besteht.

Für diesen Beitrag wurden von den Autorinnen keine Studien an Menschen oder Tieren durchgeführt. Ein Ethikvotum für die Fokusgruppendiskussionen wurde im Rahmen des Projektes HLS-MIG der Universität Bielefeld eingeholt. Die Einverständniserklärungen für die Einzelinterviews liegen ebenfalls vor.

Open Access. Dieser Artikel wird unter der Creative Commons Namensnennung 4.0 International Lizenz veröffentlicht, welche die Nutzung, Vervielfältigung, Bearbeitung, Verbreitung und Wiedergabe in jeglichem Medium und Format erlaubt, sofern Sie den/die ursprünglichen Autor(en) und die Quelle ordnungsgemäß nennen, einen Link zur Creative Commons Lizenz beifügen und angeben, ob Änderungen vorgenommen wurden.

Die in diesem Artikel enthaltenen Bilder und sonstiges Drittmaterial unterliegen ebenfalls der genannten Creative Commons Lizenz, sofern sich aus der Abbildungslegende nichts anderes ergibt. Sofern das betreffende Material nicht unter der genannten Creative Commons Lizenz steht und die betreffende Handlung nicht nach gesetzlichen Vorschriften erlaubt ist, ist für die oben aufgeführten Weiterverwendungen des Materials die Einwilligung des jeweiligen Rechteinhabers einzuholen.

Weitere Details zur Lizenz entnehmen Sie bitte der Lizenzinformation auf http://creativecommons.org/ licenses/by/4.0/deed.de.

\section{Literatur}

1. Abel T (2007) Cultural capital in health promotion In: McQueen DV, Kickbusch I, Potvin L, Pelikan JM, Balbo L, Abel T (Hrsg) Health and modernity: the role of theory in health promotion. Springer, New York, S43-73

2. Becerra MB, Becerra JB, Daus GP, Martin LR (2015) Determinants of Low Health Literacyamong AsianAmerican and Pacific Islanders in California.J Racial and Ethnic Health Disparities 2(2):267-273

3. Berens E-M, Ganahl K, Vogt D, Schaeffer D (2021) Health literacy in the domain of Healthcare among older migrants in Germany (North RhineWestphalia). Findings from a cross-sectional survey. Int J Migr Health Soc Care 17(1):62-74. https://doi.org/10.1108/IJMHSC-09-2019-0078

4. Berger F (2018) Typ-2-Diabetes und Migranten: Menschen aus verschiedenen Sprach- und Kulturräumen. Diabetol Stoffwechs 13(03):241-256

5. Bieri U, Kocher J, Gauch C, Tschöpe S, Venetz $A$, Hagemann $M$, Schwab J, Schüpbach $S$, Frind A (2016) Bevölkerungsbefragung „Erhebung Gesundheitskompetenz 2015“. Schlussbericht. gfs.bern

6. Bittlingmayer UH, Islertas Z, Sahrai E, Harsch S, Bertschi I, Sahrai D (2020) Health Literacy aus gesundheitsethnologischer Perspektive. Eine Analyse alltäglicher Gesundheitspraktiken von migrantischen Jugendlichen und Familien. Springer VS, Wiesbaden

7. Bohnsack R (2008) Rekonstruktive Sozialforschung. Einführung in qualitative Methoden 7. Aufl. Barbara Budrich, Opladen \& Farmington Hills

8. Champlin S, Hoover SD, Mackert M (2019) Family health in adult education: a missing piece of the health literacy puzzle. Health Lit Res Pract 3(Suppl):S75-S78

9. Dresing T, Pehl T (2018) Praxisbuch Interview, Transkription \& Analyse. Anleitungen und Regelsysteme für qualitativ Forschende, 8. Aufl. Eigenverlag, Marburg

10. Edwards M, Wood F, Davies M, Edwards A (2015) 'Distributed health literacy': Longitudinal qualitative analysis of the roles of health literacy mediators and social networks of people living with a longterm health condition. Health Expect 18(5):1180-1193

11. Flick U (2009) Qualitative Sozialforschung. Eine Einführung. Rowohlt, Hamburg
12. Ganahl K, Pelikan JM (2017) Gesundheitskompetenz von 15-jährigen Jugendlichen in Österreich - im Vergleich zur Gesamtbevölkerung. In: Schaeffer D, Pelikan JM (Hrsg) Health Literacy. Forschungsstand und Perspektiven. Hogrefe, Bern, S175-188

13. Geene R (2018) Familiäre Gesundheitsförderung. In: Hurrelmann K, Richter M, Klotz T, Stock S (Hrsg) Referenzwerk Prävention und Gesundheitsförderung. Grundlagen, Konzepte und Umsetzungsstrategien, 5. Aufl. Hogrefe, Göttingen, S371-338

14. Helfferich C (2004) Die Qualität qualitativer Daten. Manual für die Durchführung qualitativer Interviews. VS, Wiesbaden

15. HLS-EU Consortium (2012) Comparative report on health literacy in eight EU member states. The European health literacy project 2009-2012. Ludwig Boltzmann Institute for Health Promotion Research, Vienna

16. Kuckartz U (2012) Qualitative Inhaltsanalyse. Methoden, Praxis, Computerunterstützung. Beltz Juventa, Weinheim

17. Lampert T, Kuntz B, Hoebel J, Müters S, Kroll LE (2018) Gesundheitliche Ungleichheit. In: Statistisches Bundesamt (Destatis), Wissenschaftszentrum Berlin (Hrsg) Datenreport 2018. Ein Sozialbericht für die Bundesrepublik Deutschland. Bundeszentrale für politische Bildung, Bonn, S302-313

18. Levin-Zamir D, Leung AYM, Dodson S, Rowlands G (2017) Health literacy in selected populations: Individuals, families, and communities from the international and cultural perspective. ISU 37(2):131-151

19. Maier G, Felder-Puig R (2017) Gesundheitskompetenzen von Kindern und Jugendlichen. Herausforderungen und Überblick zum aktuellen Stand der Forschung, Wien. https://www.ifgp. at/cdscontent/load?contentid=10008.649229\& version=1518160812. Zugegriffen: 30.0 kt. 2020

20. Mayring P (2015) Qualitative Inhaltsanalyse, 12. Aufl. Beltz, Weinheim

21. Papen U (2009) Literacy, learning and health-a social practices view of health literacy. Lit Numeracy Stud 16/17(2/1):19-34

22. ParkerR (2009) Measuring health literacy: what? So what? Now what? In: Hernandez L (Hrsg) Measures of health literacy: workshop summary, roundtable on health literacy. National Academies Press, Washington DC, S91-98

23. Quenzel G, Schaeffer D (2016) Health Literacy - Gesundheitskompetenz vulnerabler Bevölkerungsgruppen. Universität Bielefeld, Bielefeld

24. Rommel A, Saß AC, Born S, Ellert U (2015) Die gesundheitliche Lage von Menschen mit Migrationshintergrund und die Bedeutung des sozioökonomischen Status: Erste Ergebnisse der Studie zur Gesundheit Erwachsener in Deutschland (DEGS1). Bundesgesundheitsblatt Gesundheitsforschung Gesundheitsschutz 58(6):543-552

25. Samerski S (2019) Health literacy as a social practice: social and empirical dimensions of knowledge on health and healthcare. Soc Sci Med 226:1-8

26. Schaeffer D, Hurrelmann K, Bauer U, Kolpatzik K (Hrsg) (2018) Nationaler Aktionsplan Gesundheitskompetenz. Die Gesundheitskompetenz in Deutschland stärken. KomPart, Berlin

27. Schaeffer D, Berens E-M, Gille S, Griese L, Klinger J, de Sombre S, Vogt D, Hurrelmann K (2021) Gesundheitskompetenz der Bevölkerung in Deutschland vor und während der Corona Pandemie. Ergebnisse des HLS-GER 2. Universität 
Bielefeld, Interdisziplinäres Zentrum für Gesundheitskompetenzforschung, Hertie School Berlin, Bielefeld Berlin

28. Schaeffer D, Vogt $D$, Berens $E-M$, Hurrelmann $K$ (2016) Gesundheitskompetenz der Bevölkerung in Deutschland: Ergebnisbericht. Universität Bielefeld, Fakultät für Gesundheitswissenschaften, Bielefeld

29. Sivanand B, Herman A, Teutsch C, Teutsch $S$ (2017) Building health literacy and family engagement in head start communities: a case study. https://nam.edu/wp-content/uploads/ 2017/04/Building-Health-Literacy-and-FamilyEngagement-in-Head-Start-Communities.pdf. Zugegriffen: 11.Nov. 2020

30. Sørensen K, Van den Broucke S, Fullam J et al (2012) Health literacy and public health: a systematic review and integration of definitions and models. BMCPublic Health 12(1):80

31. Tepecik E (2010) Bildungserfolge mit Migrationshintergrund. Biographien bildungserfolgreicher MigratnInnen türkischer Herkunft. Springer VS, Wiesbaden

32. Todd L, Hoffmann-Goetz L (2011) Predicting health literacy among English-as-a-second-language older Chinese immigrant women to Canada: comprehension of colon cancer prevention information. J Cancer Educ 26(2):326-332

33. Wångdahl J, Lytsy $P$, Martensson L, Westerling R (2014) Health literacy among refugees in Sweden-A cross-sectional study. BMC Public Health 14(1030):1-12

34. Zielke-Nadkarni A (2003) Individualpflege als Herausforderung in multikulturellen Pflegesituationen. Eine ethnografische Studie mit türkischen und deutschen Frauen. Huber, Bern

35. Statistisches Bundesamt https://www. destatis.de/DE/Themen/Gesellschaft-Umwelt/ Bevoelkerung/Migration-Integration/Glossar/ migrationshintergrund.html. Zugegriffen:29. Okt. 2020

36. Statistisches Bundesamt (2020) Bevölkerung und Erwerbstätigkeit - Bevölkerung mit Migrationshintergrund - Ergebnisse des Mikrozensus 2019. Fachserie 1 Reihe 2.2., Statistisches Bundesamt (Destatis) 